\title{
Measuring the neutron star equation of state with gravitational wave observations
}

\author{
Jocelyn S. Read, ${ }^{1}$ Charalampos Markakis, ${ }^{2}$ Masaru Shibata, ${ }^{3}$ Kōji Uryū, ${ }^{4}$ Jolien D. E. Creighton, ${ }^{2}$ and John L. Friedman ${ }^{2}$ \\ ${ }^{1}$ Max-Planck-Institut für Gravitationsphysik, Albert-Einstein-Institut, Golm, Germany \\ ${ }^{2}$ Department of Physics, University of Wisconsin-Milwaukee, P.O. Box 413, Milwaukee, WI 53201, USA \\ ${ }^{3}$ Graduate School of Arts and Sciences, University of Tokyo, Komaba, Meguro, Tokyo 153-8902, Japan \\ ${ }^{4}$ Department of Physics, University of the Ryukyus, 1 Senbaru, Nishihara, Okinawa 903-0213, Japan
}

\begin{abstract}
We report the results of a first study that uses numerical simulations to estimate the accuracy with which one can use gravitational wave observations of double neutron star inspiral to measure parameters of the neutron-star equation of state. The simulations use the evolution and initial-data codes of Shibata and Uryu to compute the last several orbits and the merger of neutron stars, with matter described by a parametrized equation of state. Previous work suggested the use of an effective cutoff frequency to place constraints on the equation of state. We find, however, that greater accuracy is obtained by measuring departures from the point-particle limit of the gravitational waveform produced during the late inspiral. As the stars approach their final plunge and merger, the gravitational wave phase accumulates more rapidly for smaller values of the neutron star compactness (the ratio of the mass of the neutron star to its radius). We estimate that realistic equations of state will lead to gravitational waveforms that are distinguishable from point particle inspirals at an effective distance (the distance to an optimally oriented and located system that would produce an equivalent waveform amplitude) of $100 \mathrm{Mpc}$ or less. As Lattimer and Prakash observed, neutron-star radius is closely tied to the pressure at density not far above nuclear. Our results suggest that broadband gravitational wave observations at frequencies between 500 and $1000 \mathrm{~Hz}$ will constrain this pressure, and we estimate the accuracy with which it can be measured. Related first estimates of radius measurability show that the radius can be determined to an accuracy of $\delta R \sim 1 \mathrm{~km}$ at $100 \mathrm{Mpc}$.

PACS numbers: 04.25.dk, 04.30.Tv, 04.25.Nx, 26.60.Kp, 04.80.Nn
\end{abstract}

\section{INTRODUCTION}

Gravitational wave observations can potentially measure properties of neutron star equations of state (EOS) by measuring departures from the point-particle approximation to the gravitational waveform produced during the late inspiral phase of binary neutron star coalescence. We examine here the accuracy with which detectors with the sensitivity of Advanced LIGO can extract from inspiral waveforms an EOS parameter associated with the stiffness of the neutron star EOS above nuclear density.

To do this, we use a set of numerical simulation waveforms produced by varying the EOS used to model the neutron star matter. The signal analysis focuses on the late inspiral, as the radius of the orbit $r$ approaches the neutron star radius $R$. The orbital dynamics in this region will depend on the radius and internal structure of the neutron star, which in turn depend on the EOS. We also estimate the accuracy with which neutron star radii, closely linked to the EOS parameter varied, can be extracted. This is a preliminary study, using a first set of multiorbit binary neutron star waveforms. Subsequent work will use an improved AMR code with higher resolution to obtain higher accuracy and to more fully explore the EOS parameter space.

The study of radius and EOS effects on gravitational wave inspiral was first aimed at questions of detectability [1, 2], showing that the tidal effects would only affect phase evolution at the end of the inspiral and that point particle waveforms could be used for template-based detection in $\mathrm{LIGO}^{1}$. Subsequently, the competing effects of relativity and finite size at the end of binary neutron star inspiral have been studied with an array of different approximations, yielding estimates of the gravitational wave spectrum which depart from that of a point particle inspiral starting somewhere between $500 \mathrm{~Hz}$ and over $1000 \mathrm{~Hz}$ [4, 5, 6, 7, 8, 9, 10, 11].

The study of EOS signature on gravitational waveforms has often focused on the merger and coalescence phases of the waveform [12, 13, 14], above the frequency of the last orbit. These frequencies are higher than the sensitive band of ground-based interferometers like Advanced LIGO, except in carefully tuned high frequency narrow-band configurations. Early work on measurability of finite size effects [15] used a model of point-particle inspiral truncated at such a frequency, which could be sought with narrow-band tuning.

The characteristic frequency where EOS effects become important for the gravitational waveform is often estimated via an innermost stable circular orbit or Roche lobe overflow in a quasiequilibrium approximation. With many such estimates above $1000 \mathrm{~Hz}$ [7, 11, 16], some summaries of neutron star radius measurability with gravitational waves have assumed that EOS dependence in double neutron-star binaries is unlikely to be detected with Advanced LIGO [17].

However, for quantitatively studying the late inspiral and merger phases of binary neutron stars, numerical relativity is required. Until quite recently, there has been no general rel-

\footnotetext{
${ }^{1}$ However with the increased sensitivity of Advanced LIGO, the contribution to phase evolution from tidal effects may affect the waveform during the early, low-frequency part of the inspiral [3].
} 
ativistic simulation that quantitatively clarifies the inspiral to merger phases primarily because of limitation of the computational resources, although a number of simulations have been done for a qualitative study [12, 13, 18, 19, 20, 21, 22, 23, 24]. The crucial drawback in the previous works was that the simulations were short-term for the inspiral phase; the inspiral motion of the neutron stars was followed only for about one orbit, and the gravitational wave spectrum determined only above $1 \mathrm{kHz}$ (but see [25, 26]). Furthermore, the simulations were usually started with a quasi-circular state in which the approaching velocity between two neutron stars is assumed to be zero and thus the eccentricity is not exactly zero. Thus, in the previous studies, the radial velocity at the onset of merger is not correctly taken into account and the non-zero eccentricity could play an unfavorable role.

In this paper, by examining longer evolutions of binary neutron stars, the early relaxation from the quasi-circular state can be removed. By comparing the numerical inspiral waveform to that of point particles, we confirm that the frequency evolution in the late inspiral differs from the point particle case, accumulating phase more quickly in the final orbits and merging at earlier times. This effect depends systematically on the EOS and resultant radius of the neutron stars simulated, and for large variations in EOS and radius the effect is larger than estimates of error in the numerical waveform.

We calculate the signal strength of this difference in waveform using the sensitivity curves of commissioned and proposed gravitational wave detectors, and find that there is a measurably different signal at reasonable distances from the inspirals of binary neutron stars with different EOS. This leads to a first quantitative estimate of the measurability of EOS with Advanced LIGO.

We also note that the broadband configuration of Advanced LIGO does as well or better as narrow band configurations in detecting the difference between neutron star EOS. An improved understanding of the sensitivity of different gravitational wave detector configurations to neutron star structure will be essential for the design of next-generation detectors for gravitational wave astrophysics.

\section{EQUATIONS OF STATE}

We choose EOS based on work done in [27] to develop a parameterized EOS that accurately reproduces features of realistic EOS. Systematic variation of the EOS parameters allows us to determine which properties significantly affect the gravitational radiation produced, and thus can be constrained with sufficiently strong gravitational wave detections.

The models chosen for this study use a variation of one EOS parameter in the neutron star core. The EOS pressure $p$ is specified as a function of rest mass density ${ }^{2}$. Rest mass

\footnotetext{
${ }^{2}$ Rest mass density $\rho=m_{\mathrm{b}} n$ is proportional to number density $n$ with the mass per baryon, if the matter were to be dispersed to infinity, fixed to be $m_{\mathrm{b}}=1.66 \times 10^{-24} \mathrm{~g}$.
}

FIG. 1: Initial choices of EOS for numerical evolution compared to the set of tabled EOS considered in [27]. Candidates are labelled in order of increasing softness: $2 \mathrm{H}, \mathrm{H}, \mathrm{HB}, \mathrm{B}, 2 \mathrm{~B}$.

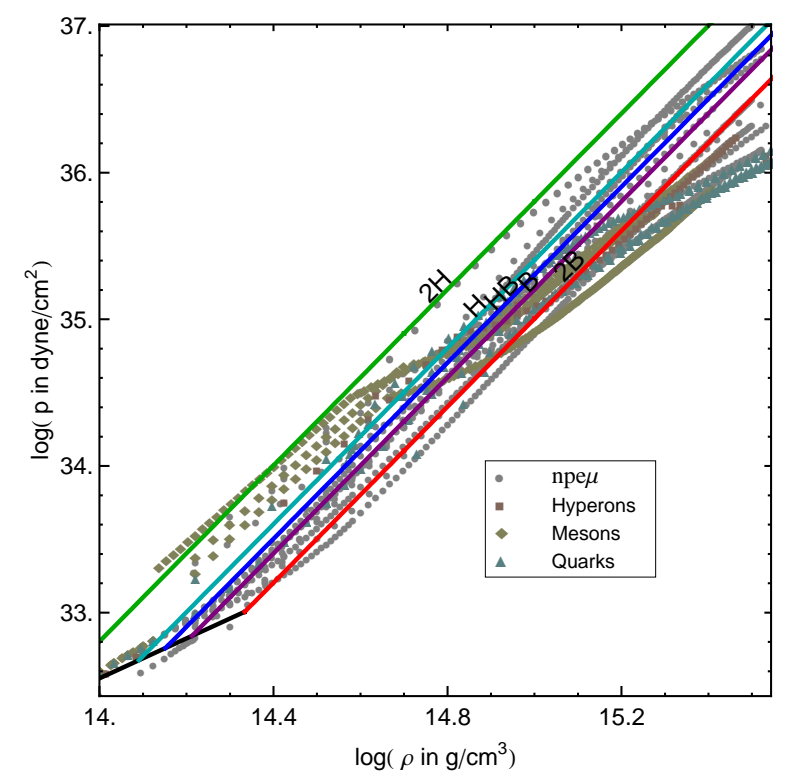

density $\rho$, and the resulting energy density $\epsilon$ are then determined by the first law of thermodynamics. We use piecewise polytropic EOS, of the form

$$
p(\rho)=K_{i} \rho^{\Gamma_{i}}, \quad d \frac{\epsilon}{\rho}=-p d \frac{1}{\rho},
$$

in a set of intervals $\rho_{i} \leq \rho \leq \rho_{i+1}$ in rest mass density, with $\epsilon / \rho \rightarrow 1$ as $\rho \rightarrow 0$.

A fixed crust EOS models the behavior of matter down to $10^{12} \mathrm{~g} \mathrm{~cm}^{-3}$; the numerical simulations considered do not resolve densities below this. The crust is modelled with a single polytrope region, fitted to tabulated crust EOS, for the region above neutron drip. The polytrope has $\Gamma_{\text {crust }}=1.3569$, with $K_{\text {crust }}$ chosen so that $p / c^{2}=1.5689 \times 10^{31} \mathrm{dyn} \mathrm{cm}^{-2}$ when $\rho=10^{13} \mathrm{~g} \mathrm{~cm}^{-3}$. The core EOS is constructed independently of crust behavior, and the dividing density between the crust and core varies by EOS.

In [27] it was found that three zones within the core are needed to accurately model the full range of proposed EOS models; however in this paper we will consider only one core zone, described by just one polytropic EOS. We vary the core EOS with an overall pressure shift $p_{1}$, specified at the fiducial density $\rho_{1}=5.0119 \times 10^{14} \mathrm{~g} \mathrm{~cm}^{-3}$, while holding the adiabatic index in all regions of the core fixed at $\Gamma=3$. While only a subset of realistic EOS are well-approximated by a single core polytrope, reducing the EOS considered to this single-parameter family allows us to estimate parameter measurability with a reasonable number of simulations.

After fixing the core adiabatic index, increasing the overall pressure scale $p_{1}$ produces a family of neutron stars with progressively increasing radius for a given mass; the $p_{1}$ parameter 
TABLE I: Properties of initial EOS. These range from the "softest" EOS at the top, which results in a prompt collapse to a black hole upon merger, to the "hardest" (or "stiffest") at the bottom. Model HB is considered a typical EOS. The pressure $p_{1}$, which is the pressure at density $\rho_{1}=5 \times 10^{14} \mathrm{~g} \mathrm{~cm}^{-3}$, determines the polytropic EOS for the neutron star core; all candidates have $\Gamma=3$. Radius $R$ and compactness $G M / c^{2} R$ are those of a single isolated $M=1.35 M_{\odot}$ TOV star where radius is measured in Schwarzschild-like coordinates. An astrophysically important EOS-dependent parameter is the maximum neutron star mass, $M_{\max }$, which is given in the fifth column.

\begin{tabular}{lcrcc}
\hline Model $\log _{10} p_{1}\left[\mathrm{dyn} \mathrm{cm}^{-2}\right]$ & $R[\mathrm{~km}]$ & $G M / c^{2} R$ & $M_{\max }\left[M_{\odot}\right]$ \\
\hline \hline 2H & 34.90 & 15.2 & 0.13 & 2.83 \\
$\mathrm{H}$ & 34.50 & 12.3 & 0.16 & 2.25 \\
$\mathrm{HB}$ & 34.40 & 11.6 & 0.17 & 2.12 \\
$\mathrm{~B}$ & 34.30 & 10.9 & 0.18 & 2.00 \\
2B & 34.10 & 9.7 & 0.21 & 1.78 \\
\hline
\end{tabular}

was chosen in part because Lattimer and Prakash [28] found that pressure at one to two times nuclear density is closely tied to neutron star radius, with $R \propto p_{1}^{1 / 4}$. The radius is less sensitive to variation of the adiabatic index in the neutron star core, for reasonable adiabatic indices [27].

An important element of future work will be incorporating additional variations of the EOS within the core. This could involve additional models of varying adiabatic index around a fixed $p_{1}$, as well as multiple piecewise-polytrope zones within the core or EOS parameters yielding neutron stars of the same $R$ but different internal structure. Such work would yield insight into the relative size and correlation of effects on the orbital evolution due to the stellar radius and internal structure.

The first models were chosen with EOS that "bracket" the range of existing candidates, seen in Fig. 1] These models are HB with $p_{1}=10^{34.40} \mathrm{dyn}^{-2}$, a standard EOS; $2 \mathrm{H}$ with $p_{1}=10^{34.90} \mathrm{dyn} \mathrm{cm}^{-2}$, a stiff EOS; 2B, with $p_{1} / c^{2}=10^{34.10}{\mathrm{dyn} \mathrm{cm}^{-2}}^{-}$, a soft EOS. Additional models B with $p_{1} / c^{2}=10^{34.30} \mathrm{dyn} \mathrm{cm}^{-2}$ and $\mathrm{H}$ with $p_{1} / c^{2}=$ $10^{34.50} \mathrm{dyn} \mathrm{cm}^{-2}$, were chosen with small shifts in parameter from HB to better estimate local parameter dependence of the waveform.

\section{NUMERICAL METHODS}

For each EOS considered, we simulate the late inspiral and merger of a binary neutron star system. For this study, we fix the gravitational mass of each neutron star in the binary to $1.35 M_{\odot}$, an average value for pulsars observed in binary systems [29, 30]. We expect the significance of tidal effects in this configuration to be fairly representative of tidal effects over the relatively narrow range of masses and mass ratios expected in astrophysical binary neutron star system.

\section{A. Initial data}

Conformally flat initial data is generated by constructing a quasiequilibrium sequence of irrotational neutron stars in binary system, following the methods of [7, 16, 31, 32, 33, 34]. As in previous work, we assume irrotational flow fields, neglecting spin of the neutron stars. This assumption is based on the estimation of negligible tidal spin-ups [1, 2]. The parameterized EOS of Eq. (1) is incorporated in the code to solve for initial data with a conformally flat spatial geometry, using the Isenberg-Wilson-Mathews formulation [35, 36] coupled to the neutron star matter equation consistently. Each of the stars has a baryon number equal to that of an isolated star with gravitational mass $M=1.35 M_{\odot}$. The initial data for the full numerical simulation is taken from the quasi-equilibrium configuration at a separation such that $\sim 3$ orbits remain before merger. Relevant quantities of the initial configurations for each parameterized EOS are presented in Table II

\section{B. Numerical evolution}

The Einstein equations are evolved with the original version of the Baumgarte-Shapiro-Shibata-Nakamura formulation [37, 38] in which we evolve the conformal factor, $\varphi=$ $(\ln \gamma) / 12$, the trace $K$ of the extrinsic curvature, the conformal three metrics, $\tilde{\gamma}_{i j} \equiv \gamma^{-1 / 3} \gamma_{i j}$, the tracefree part of the extrinsic curvature, $\tilde{A}_{i j} \equiv \gamma^{-1 / 3}\left(K_{i j}-K \gamma_{i j} / 3\right)$, and an auxiliary three-vector, $F_{i} \equiv \delta^{j k} \partial_{j} \tilde{\gamma}_{i k}$. Here $\gamma_{i j}$ is the three metric, $K_{i j}$ the extrinsic curvature, $\gamma \equiv \operatorname{det}\left(\gamma_{i j}\right)$, and $K \equiv K_{i j} \gamma^{i j}$. As in [39], we evolve the conformal factor $\varphi$, not the inverse of $\psi$, because the cell-centered grid is adopted in our code, and hence, the black hole spacetime is handled in the moving puncture framework [40, 41]. For the conditions on the lapse, $\alpha$, and the shift vector, $\beta^{i}$, we adopt a dynamical gauge condition as in [39].

The numerical scheme for solving the Einstein equation is the same as that in [39]: We use the fourth-order finite difference scheme in the spatial direction and a third-order Runge-Kutta scheme in the time integration, where the advection terms such as $\beta^{i} \partial_{i} \varphi$ are evaluated by a fourth-order non-centered difference.

The hydrodynamics equations are solved as in [39]: We evolve $\rho_{*} \equiv \rho \alpha u^{t} e^{6 \varphi}, \hat{u}_{i} \equiv h u_{i}$, and $e_{*} \equiv \rho \alpha u^{t}-P /\left(\rho \alpha u^{t}\right)$, where $\rho$ is the rest-mass density, $u_{i}$ is the three-component of the four velocity, $P$ is the pressure, and $h$ is the specific enthalpy defined by $h \equiv 1+\varepsilon+P / \rho$ and $\varepsilon$ is the specific internal energy defined by $\varepsilon \equiv \epsilon / \rho-1$. To handle advection terms in the hydrodynamic equations, a high-resolution central scheme [42] is adopted with a third-order piecewise parabolic interpolation and with a steep min-mod limiter. In the present work, the limiter parameter, $b$, is set to be 2.5 (see [43] for detail about the parameter $b$ ).

The fluid evolution during inspiral is essentially free of shocks, and when there are no shocks the simulations use the cold EOS specified in Sec. II. During merger, when the evolution has shocks, we include a hot component with a thermal effective adiabatic index $\Gamma_{\mathrm{i}}$, as described in [43]. Shock heat- 
TABLE II: Quantities of each initial data set for irrotational binary neutron stars. Each star has a baryon number $M_{0}$ equal to that of an isolated $M=1.35 M_{\odot}$ star. The ADM mass $M_{\mathrm{ADM}}$ of the initial slice includes the binding energy, $J$ is the total angular momentum of the initial slice. The binary compactness $C_{0}$ is defined by $C_{0}=\left(\Omega M_{\mathrm{ADM}} G c^{-3}\right)^{2 / 3}$.

\begin{tabular}{lcccccc}
\hline Model & $\rho_{\max }\left[\mathrm{g} \mathrm{cm}^{-3}\right]$ & $M_{0}\left[M_{\odot}\right]$ & $M_{\text {ADM }}\left[M_{\odot}\right]$ & $c J /\left(G M_{\text {ADM }}^{2}\right)$ & $\Omega / 2 \pi[\mathrm{Hz}]$ & $C_{0}$ \\
\hline \hline 2H & $3.73196 \times 10^{14}$ & 1.45488 & 2.67262 & 0.993319 & 324.704 & $8.96966 \times 10^{-2}$ \\
H & $7.02661 \times 10^{14}$ & 1.48385 & 2.67080 & 0.989524 & 321.468 & $8.90593 \times 10^{-2}$ \\
HB & $8.27673 \times 10^{14}$ & 1.49273 & 2.67290 & 0.995361 & 309.928 & $8.69582 \times 10^{-2}$ \\
B & $9.77811 \times 10^{14}$ & 1.50247 & 2.67290 & 0.992638 & 314.170 & $8.77522 \times 10^{-2}$ \\
2B & $1.38300 \times 10^{15}$ & 1.52509 & 2.67229 & 0.987681 & 321.170 & $8.90375 \times 10^{-2}$ \\
\hline
\end{tabular}

ing in merger can increase the thermal energy up to $\sim 20-30 \%$ of the total energy [12].

Gravitational radiation is extracted both by spatially decomposing the metric perturbation about flat spacetime in the wave-zone with spin-2 weighted spherical harmonics and by calculating the outgoing part of the Weyl scalar $\Psi_{4}$. For equal mass neutron stars (such as those studied here), the quadrupole $(\ell=2, m= \pm 2$ ) mode is much larger than any other mode; we consider just this mode in this analysis.

The waveforms output from the simulations are the cross and plus amplitudes $h_{+} c^{2} D / G M_{\text {tot }}$ and $h_{\times} c^{2} D / G M_{\text {tot }}$ of the quadrupole waveform, as would be measured at large distance $D \gg G M_{\text {tot }} / c^{2}$ along the $z$ axis perpendicular to the plane of the orbit, versus the retarded time $t_{\text {ret }}$. Here $M_{\text {tot }}$ is the sum of the two neutron star masses when they are far apart, $M_{\text {tot }}=2.7 M_{\odot}$. The strain is sampled at discrete values evenly spaced in $t$, with a sampling rate $\Delta t$ of between $0.006 \mathrm{~ms}$ (for $2 \mathrm{~B}$ ) and $0.031 \mathrm{~ms}$ (for $2 \mathrm{H}$ ) which depends on the time of simulation. There is junk radiation in the early part of the extracted waveforms for $t_{\text {ret }} \lesssim 0$. We discard this part in the data analysis.

Each simulation is typically performed for three grid resolutions. In the best-resolution case, the diameter of neutron stars is covered by 60 grid points. Convergence tests with different grid resolutions indicate that, with the best grid resolution, the time duration in the inspiral phase is underestimated by about 1 orbit. This is primarily due to the fact that angular momentum is spuriously lost by numerical dissipation. Thus, the inspiral gravitational waves include a phase error, and as a result, the amplitude of the spectrum for the inspiral phase is slightly underestimated. However, we find that the waveforms and resulting power spectrum for the late inspiral and merger phases, which we are most interested in for the present work, depend weakly on the grid resolution.

\section{WAVEFORM ANALYSIS}

We construct the complex quantity

$$
h=h_{+}-i h_{\times}
$$

from the quadrupole waveform data. The amplitude and phase of this quantity define the instantaneous amplitude $|h|$ and phase $\phi=\arg h$ of the waveform. The instantaneous fre- quency $f$ of the quadrupole waveform is then estimated by

$$
f=\frac{1}{2 \pi} \frac{\Delta \phi}{\Delta t}
$$

The numerical data can be shifted in phase and time by adding a time shift $\tau$ to the time series points and multiplying the complex $h$ by $e^{i \phi}$ to shift the overall wave phase by $\phi$.

It is useful to define a reference time marking the end of the inspiral and onset of merger. A natural choice for the end of the inspiral portion, considering the behaviour of a PP inspiral waveform, is the time of the peak in the waveform amplitude $|h|$. However, the amplitude of the numerical waveforms oscillates over the course of an orbit. A moving average of the waveform amplitude over $0.5 \mathrm{~ms}$ segments is used to average this oscillation; the end of the inspiral is then defined as the time at the end of the maximum amplitude interval. The resulting merger time $t_{\mathrm{M}}$ will be marked by solid vertical lines in the plots to follow.

The numerical waveforms begin at different orbital frequencies. To align them for comparison, they are each matched in the early inspiral region to the same postNewtonian point-particle (or PP) waveform.

\section{A. Post-Newtonian point particle}

In full GR, point-particle inspiral is not well-defined, and one is left with the post-Newtonian point-particle (PP) approximation and fully general relativistic black-hole numerical solutions as natural substitutes. Fortunately, the Taylor T4 3.0/3.5 post-Newtonian specification, introduced in [44], agrees closely with numerical binary black hole waveforms for many cycles, up to and including the cycle before merger (see also [45]). This empirical agreement allows us to adopt the Taylor T4 waveform as an appropriate PP baseline waveform, compatible with full GR until the last cycles. We will show that the binary neutron star waveforms depart from this waveform 4-8 cycles (200-560 $M_{\text {tot }}$ ) before the best-fit PP merger.

The TaylorT4 waveform is constructed by numerically integrating for a post-Newtonian parameter $x$, which is related to the orbital frequency observed at infinity $\Omega$ :

$$
x=\left(\frac{G M_{\mathrm{tot}} \Omega}{c^{3}}\right)^{2 / 3},
$$


FIG. 2: Solid lines show numerical waveforms, scaled by $c^{2} D / G M_{\text {tot }}$, and aligned in time and phase to the same point-particle post-Newtonian inspiral (dashed line), using the method described in Sec. IVB The two dashed vertical bars indicate the portion of the waveform used for matching; the last vertical bar indicates the end of inspiral time $t_{\mathrm{M}}$ for the numerical waveform. The top four simulations, $2 \mathrm{H}, \mathrm{H}, \mathrm{HB}$, and $\mathrm{B}$, show the start of post-merger oscillations from a hypermassive NS remnant in the simulation. $2 \mathrm{~B}$ shows quasinormal ringdown from a prompt collapse to a black hole following merger.
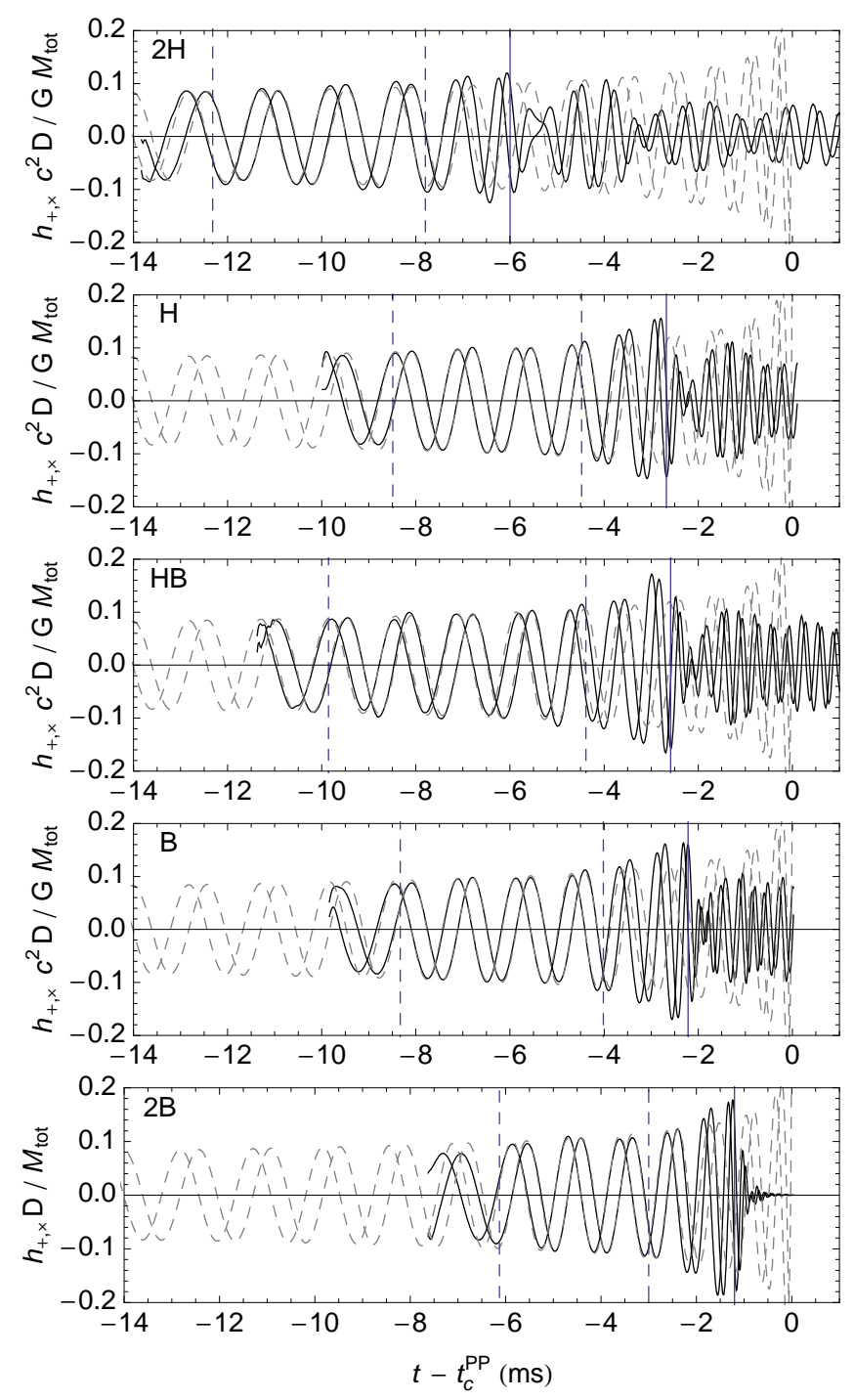

where $M_{\text {tot }}$ is the sum of the point masses. To first postNewtonian order, $x \sim M_{\text {tot }} / r$ where $r$ is the orbital radius.
Following [44], we use

$$
\begin{aligned}
\frac{d x}{d t}= & \frac{16}{5} \frac{c^{3}}{G} \frac{x^{5}}{M_{\mathrm{tot}}}\left[1-\frac{487}{168} x+4 \pi x^{3 / 2}+\frac{274229}{72576} x^{2}\right. \\
& -\frac{254}{21} \pi x^{5 / 2}+\left\{\frac{178384023737}{33530011200}+\frac{1475}{192} \pi^{2}\right. \\
& \left.\left.-\frac{1712}{105} \gamma-\frac{856}{105} \ln (16 x)\right\} x^{3}+\frac{3310}{189} \pi x^{7 / 2}\right],
\end{aligned}
$$

to find $x(t)$ and then orbital phase of the binary is found from

$$
\frac{d \Phi}{d t}=\frac{c^{3}}{G M_{\mathrm{tot}}} x^{3 / 2}
$$

This yields a result to 3.5 post-Newtonian order for the phase evolution. The constants of integration are fixed by specifying the coalescence time $t_{\mathrm{c}}^{\mathrm{PP}}$, when $x \rightarrow \infty$, and the orbital phase at this time $\Phi\left(t_{\mathrm{c}}^{\mathrm{PP}}\right)=\Phi_{\mathrm{c}}^{\mathrm{PP}}$,

The amplitude of the quadrupole waveform is calculated to 3.0 order in [46]. For the complex waveform $h_{\mathrm{PP}}$ measured along the orbital axis of the binary, using the appropriate spherical harmonic conventions, the result is

$$
\begin{aligned}
h_{\mathrm{PP}}= & -\frac{G}{c^{2}} \frac{M_{\text {tot }}}{D} e^{2 i \Phi} x\left[1-\frac{373}{168} x+2 \pi x^{3 / 2}-\frac{62653}{24192} x^{2}\right. \\
& -\left\{\frac{197}{42} \pi+6 i\right\} x^{5 / 2}+\left\{\frac{43876092677}{1117670400}+\frac{99}{128} \pi^{2}\right. \\
& \left.\left.-\frac{856}{105} \gamma-\frac{1712}{105} \ln (2)-\frac{428}{105} \ln (x)+\frac{428}{105} i \pi\right\} x^{3}\right]
\end{aligned}
$$

where $D$ is the distance to the observer, and logarithmic terms with a frequency scale have been absorbed into the the phase. The coalescence phase of the quadrupole point particle waveform, $\phi_{\mathrm{c}}^{\mathrm{PP}}=\arg h_{\mathrm{PP}}\left(t_{\mathrm{c}}^{\mathrm{PP}}\right)$, is determined by a choice of the orbital coalescence phase $\Phi_{\mathrm{c}}^{\mathrm{PP}}$.

\section{B. Match to post-Newtonian point particle}

To match the numerical data to the PP inspiral waveform, a relative time shift and a relative phase shift must be specified by varying these parameters to obtain the best match. The masses of the point particles in the PP waveform are fixed to be the same as the neutron stars in the numerical simulations - the gravitational mass of isolated (TOV) neutron stars with the same number of baryons - and so masses are not varied in finding the best match. With a goal of signal analysis, we choose the time and phase shift by maximizing a correlationbased match between two waveforms.

The complex numerical relativity derived quadrupole waveform, $h_{\mathrm{NR}}=h_{+}^{\mathrm{NR}}-i h_{\times}^{\mathrm{NR}}$, is convolved with the complex postNewtonian quadrupole waveform, $h_{\mathrm{PP}}=h_{+}^{\mathrm{PP}}-i h_{\times}^{\mathrm{PP}}$ of Eq. 77 , over a given matching region $T_{\mathrm{I}}<t<T_{\mathrm{F}}$.

$$
z\left(\tau ; T_{\mathrm{I}}, T_{\mathrm{F}}\right)=\int_{T_{\mathrm{I}}}^{T_{\mathrm{F}}} h_{\mathrm{NR}}(t) h_{\mathrm{PP}}^{*}(t-\tau) d t .
$$


FIG. 3: Phase-optimized, time-limited match between numerical inspiral waveforms and point particle post-Newtonian waveforms. Contours are shown at $0.95,0.98,0.99$, and 0.997. as a function of match region truncation at some time before numerical merger, $T_{\mathrm{F}}-t_{\mathrm{M}}$, and relative shift between numerical and point particle waveforms, $t_{\mathrm{c}}^{\mathrm{PP}}-t_{\mathrm{M}}$. The start of the match is fixed to $1.5 \mathrm{~ms}$ after the start of the numerical waveform. Subsequent analysis in this paper is done using the best match at a fixed $T_{\mathrm{F}}-t_{\mathrm{M}}$ of $1.8 \mathrm{~ms}$ for each waveform.
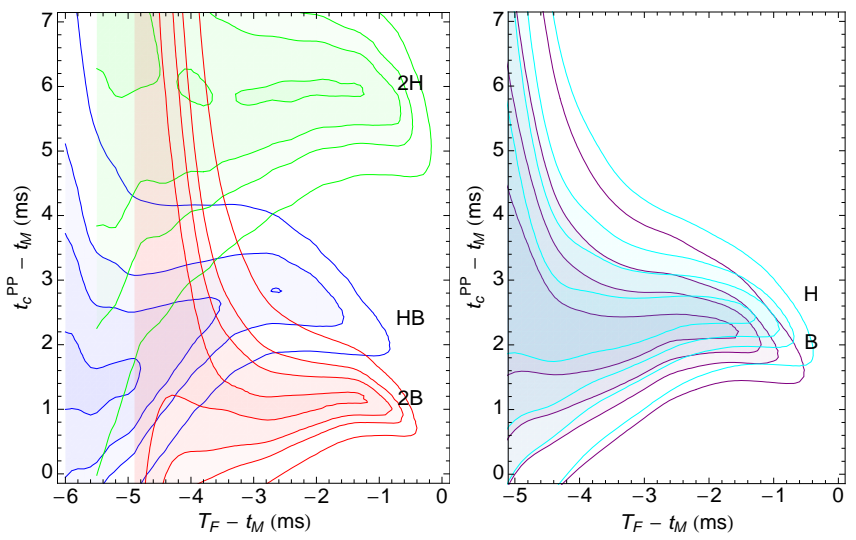

This quantity is similar to the complex matched filter output of the FINDCHIRP algorithm [47]. The real part of $z\left(\tau ; T_{\mathrm{I}}, T_{\mathrm{F}}\right)$ corresponds to the correlation of the two waveforms as a function of the time shift $\tau$. The absolute value $\left|z\left(\tau ; T_{\mathrm{I}}, T_{\mathrm{F}}\right)\right|$ is the correlation maximized over a constant overall phase shift, and the argument is the overall phase shift required to so maximize. A normalized match between two waveforms is

$$
m\left(\tau ; T_{\mathrm{I}}, T_{\mathrm{F}}\right)=\frac{z\left(\tau ; T_{\mathrm{I}}, T_{\mathrm{F}}\right)}{\sigma_{\mathrm{NR}}\left(0 ; T_{\mathrm{I}}, T_{\mathrm{F}}\right) \sigma_{\mathrm{PP}}\left(\tau ; T_{\mathrm{I}}, T_{\mathrm{F}}\right)}
$$

where the normalizing constants are defined by

$$
\sigma^{2}\left(\tau ; T_{\mathrm{I}}, T_{\mathrm{F}}\right)=\int_{T_{\mathrm{I}}}^{T_{\mathrm{F}}}|h(t-\tau)|^{2} d t
$$

where $h$ is either $h_{\mathrm{NR}}$, which defines $\sigma_{\mathrm{NR}}\left(\tau ; T_{\mathrm{I}}, T_{\mathrm{F}}\right)$, or $h_{\mathrm{PP}}$, which defines $\sigma_{\mathrm{PP}}\left(\tau ; T_{\mathrm{I}}, T_{\mathrm{F}}\right)$.

The shift $\tau$ maximizing the match between two waveforms is sensitive to the portion of the numerical waveform matched, $T_{\mathrm{I}}<t<T_{\mathrm{F}}$. Some truncation of the numerical waveform is required to eliminate residual effects of initial data. One would also like to truncate the waveform at some point before the peak amplitude, at the end of the region without significant finite size effects. To determine where this region is, we plot the match between numerical waveforms as a function of both the end point of the match $T_{\mathrm{F}}$, and the shift $\tau$, measuring quantities relative to the previously defined merger time $t_{\mathrm{M}}$ of each numerical waveform and the PP coalescence time $t_{\mathrm{c}}^{\mathrm{PP}}$ so that $\tau=t_{\mathrm{c}}^{\mathrm{PP}}-t_{\mathrm{M}}$, in Fig. 3 .

Reassuringly, for most of the waveforms, once we truncate a millisecond or so before the numerical merger $t_{\mathrm{M}}$, there is a region where the range of well-matched shifts $\tau$ show little dependence on the exact end point $T_{\mathrm{F}}$. This continues with earlier end points until the segment of the waveform considered becomes so short that the range of matching $\tau$ broadens significantly. The exception is the waveform of HB, which was the first simulation of the series. HB exhibits somewhat larger eccentricity, and drift in the best match timeshift, compared to the other waveforms.

For subsequent analysis, we choose to take $\tau$ maximizing the match for a region of the numerical waveforms between $1.5 \mathrm{~ms}$ after the start of the waveform and $1.8 \mathrm{~ms}$ before numerical merger. Varying the details of this choice can change the best match $\tau$ by up to $\simeq 1 \mathrm{~ms}$. By comparison, one waveform cycle takes between 0.5 and $2 \mathrm{~ms}$ in the inspiral region, so this will be a significant source of uncertainty in SNR estimates. Longer or more accurate simulations are required to more precisely fix a post-Newtonian match.

\section{Comparison of waveforms}

Unlike the case of matching binary black hole simulations to point particle post-Newtonian [44, 45], the binary neutron star simulations show departure from point particle many cycles before the post-Newtonian merger time. Fig. 2 shows the four waveforms shifted so the best-match PP waveforms have the same $t_{\mathrm{c}}^{\mathrm{PP}}$ and $\phi_{\mathrm{c}}^{\mathrm{PP}}$. As the stiffness of the EOS and thus the radius of the neutron stars, increases, the end of inspiral for the binary neutron stars is shifted away from the end of inspiral for point particle post-Newtonian.

This can also be seen by plotting the instantaneous frequency of the numerical simulation waveform with the same time shifts, as in Fig. 4, which also shows more clearly the difference in the post-merger oscillation frequencies of the hyper-massive remnants, when present. The larger neutron star produced by the stiff EOS $2 \mathrm{H}$ has a lower oscillation frequency than that from the medium EOS HB. The remnant forms with a bar-mode oscillation stable over a longer period than the $\sim 10 \mathrm{~ms}$ simulated. The signal from such a bar mode may be even stronger when the full lifetime is included, although aspects of the physics neglected in this study will likely come into play. Information that can be extracted from the presence (or absence) and characteristics of a post-merger oscillation signal would complement the information present in the late inspiral. This is another subject for future study.

\section{Frequency spectrum of waveforms}

Given $h_{+}$or $h_{\times}$, one can construct the discrete Fourier transforms (DFTs) $\tilde{h}_{+}$or $\tilde{h}_{\times}$. Both polarizations yield the same DFT amplitude spectrum $|\tilde{h}|$, with phase shifted by $\pi / 2$, if one neglects discretization, windowing, and numerical effects (including eccentricity). The amplitude spectrum $|\tilde{h}|$ is independent of phase and time shifts of the waveform.

The stationary phase approximation is valid for the postNewtonian waveform up to frequencies of about $1500 \mathrm{~Hz}$ (with $\lesssim 10 \%$ error), so is used to plot the amplitude of the point particle spectrum. In terms of an instantaneous fre- 
FIG. 4: Time-frequency behavior, vertical line markings as previous figure. The departure from the point particle time-frequency relations, shown using a long-dashed line, occurs between $700-1000 \mathrm{~Hz}$ depending on EOS.
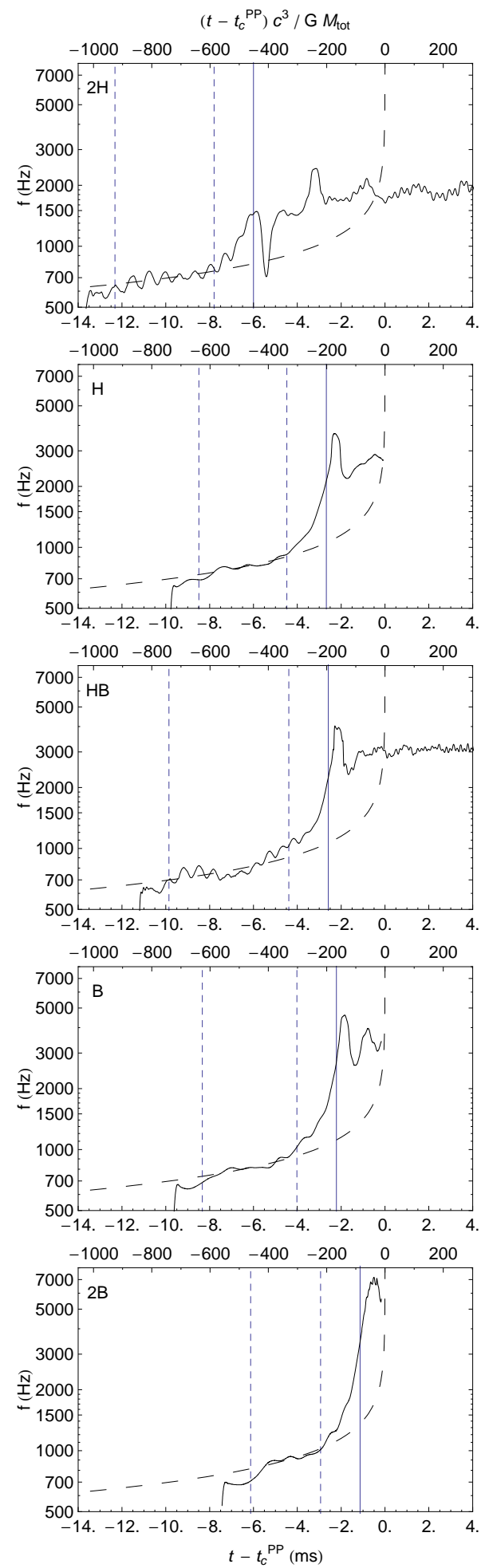

quency

$$
f(t)=\frac{1}{2 \pi} \frac{d \phi}{d t}
$$

the Fourier transform of the waveform has the amplitude

$$
|\tilde{h}| \simeq A(f)\left(\frac{d f}{d t}\right)^{-1 / 2} .
$$

For binaries comprised of equal mass companions, the gravitational radiation is dominated by quadrupole modes throughout the inspiral. The wave phase $\phi$ is negligibly different from twice the orbital phase $2 \Phi$ until the onset of merger at very high frequencies so we can use the relation

$$
\frac{d f}{d t} \simeq \frac{1}{\pi} \frac{c^{3}}{G M} \frac{3}{2} x^{1 / 2} \frac{d x}{d t}
$$

to write the amplitude of the Fourier transform entirely in terms of the functions $d x / d t$ of Eq. (5) and the amplitude of the polarization waveforms $A(f)=|h|$ in Eq. (7).

The translation of emitted waveforms into the strain amplitude measured at a detector involves transformations incorporating the effects of the emitting binary's angle of inclination and sky location. These effects are absorbed into an effective distance $D_{\text {eff }}$, which is equal to the actual distance $D$ for a binary with optimal orientation and sky location, and is greater than the actual distance for a system that is not optimally oriented or located. The detector will detect a single polarization of the waveform, some combination of the plus and cross polarizations of the emitted waveform. The polarizations extracted from the simulation can be used as two estimates of the strain at the detector for a given numerically modelled source, which give very close results and are subsequently averaged.

To compare with noise curves in the usual units, the quantity $f^{1 / 2}|h(f)|$ is plotted, at a reference distance of $D_{\text {eff }}=$ $100 \mathrm{Mpc}$ and rescaling from previously plotted numerical output $h(t) c^{2} D / G M_{\text {tot }}$ using $M_{\text {tot }}=2.7 M_{\odot}$.

The full spectra of models $2 \mathrm{H}, \mathrm{H}, \mathrm{HB}$, and B, seen in Fig. 5. show peaks at post-merger oscillation frequencies; those of $H$ and $\mathrm{B}$ are weaker as the waveform is truncated shortly after the formation of the hyper-massive remnant. Waveforms of $2 \mathrm{H}$ and $\mathrm{HB}$ are also truncated while the post-merger oscillation is ongoing; if the simulations were allowed to continue, these peaks would presumably grow further. The simulation of $2 \mathrm{~B}$, in contrast, collapses to a black hole and has only a short lived (and relatively small-amplitude) quasinormal mode ringdown post merger.

Note that time-frequency plots like the ones shown Fig. 4 showed that numerical waveforms follow the PP waveform at instantaneous frequencies of up to $700-1000 \mathrm{~Hz}$, depending on the EOS. The disagreement in the spectra in Fig. 5 from the PP stationary-phase approximation waveform at frequencies below this is primarily due to the finite starting time of the numerical waveforms. To estimate spectra from the full inspiral, we construct hybrid waveforms. The short-term numerical waveforms are are smoothly merged on to long-inspiral PP-PN waveforms with Hann windowing to smoothly turn on

$$
w(n)=\frac{1}{2}\left[1-\cos \left(\frac{\pi n}{N-1}\right)\right]
$$


FIG. 5: DFT of full numerical waveforms, at an effective distance $D_{\text {eff }}=100 \mathrm{Mpc}$, compared to noise spectra for Advanced LIGO (labelled "AdvLIGO" for the standard configuration and "Broadband" for the broad-band configuration) and the Einstein Telescope (labelled "ET") shown by thick grey lines. The DFT of the numerical waveforms turned off after $t_{\mathrm{M}}$ is shown by dot-dashed lines, the stationary-phase point particle is shown by a dashed line for reference. The lower right figure shows a combined plot of inspiral-truncated waveforms, smoothly joined on to best-match PP inspiral time series before the DFT is taken.
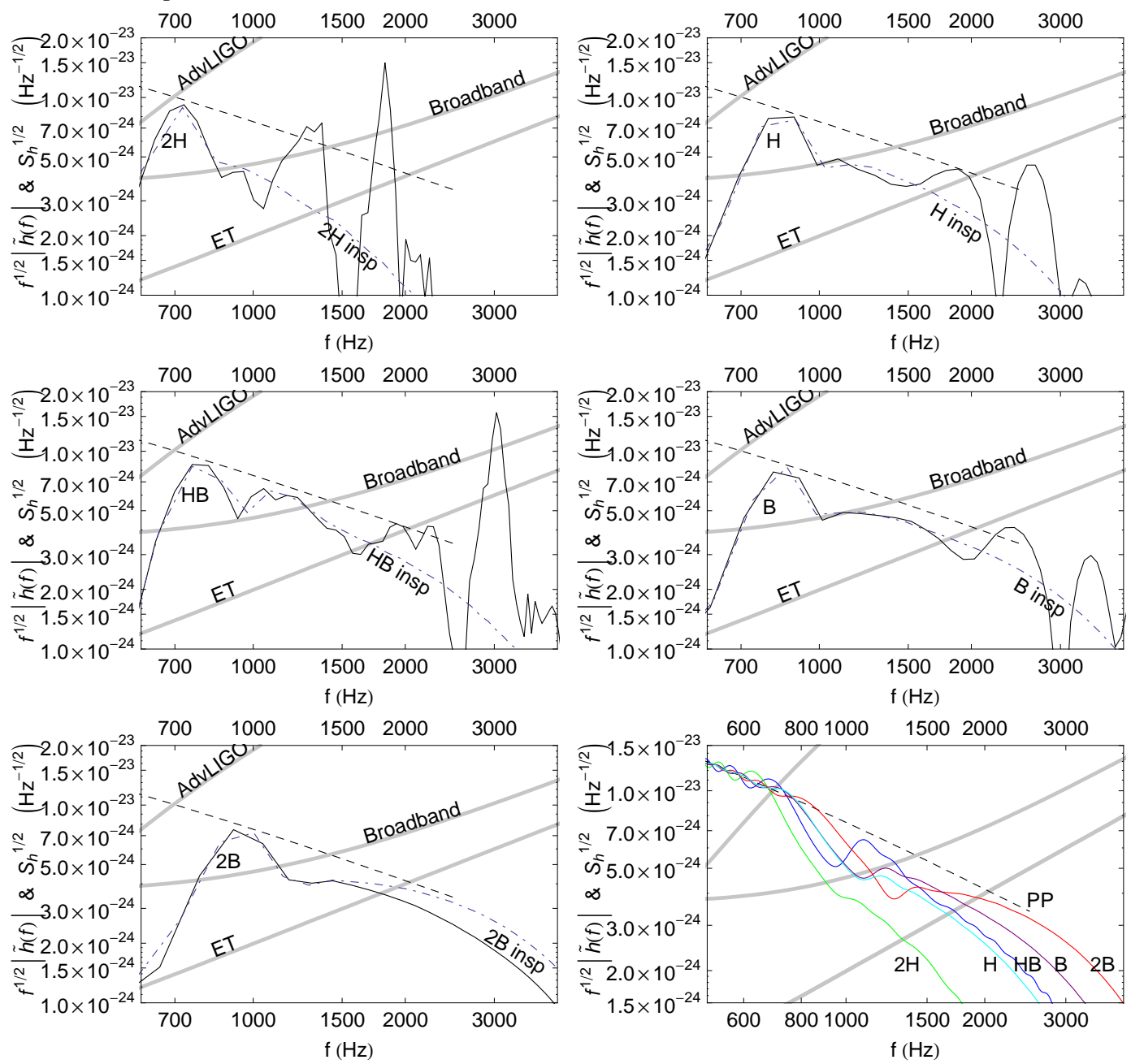

or turn off

$$
w(n)=\frac{1}{2}\left[1+\cos \left(\frac{\pi n}{N-1}\right)\right]
$$

a signal over a range of $N$ points, $0 \leq n<N$ (or over a time $N \Delta t$ ). To construct hybrid waveforms, these windows are used to turn on the numerical waveform as the matched postNewtonian is turned off, such that the sum of the two window functions is 1 over the matching range.

Although the post-merger oscillations are an interesting source of potentially measurable strains, the dependence on the cold EOS is less straightforward as temperature effects become significant during the the merger. We focus instead on the signal from the waveforms during the inspiral region only, turning off the waveforms after the end of inspiral, $t_{\mathrm{M}}$. The DFT amplitudes of inspiral-only hybrid waveforms are also plotted in Fig. 5 from the range of EOS considered. We see here that $\mathrm{HB}$ is estimated to depart from PP earlier than either

$\mathrm{H}$ or $\mathrm{B}$, rather than at the expected intermediate value. This is ascribed to the higher eccentricity and lower accuracy of the match for the HB waveform.

\section{DETECTABILITY}

The question for neutron star astrophysics is whether these differences in the waveform will be measurable. We consider the possibility of detecting EOS effects with Advanced LIGO style detectors in varying configurations.

We use several detector configurations commonly considered for Advanced LIGO include tunings optimized for $1.4 M_{\odot}$ NS-NS inspiral detection ("Standard"), for burst detection ("Broadband"), and for pulsars at $1150 \mathrm{~Hz}$ ("Narrowband"). The sensitivity is expressed in terms of the one-sided strain-equivalent amplitude spectral density $S_{h}(f)$ (which has units of $\mathrm{Hz}^{-1 / 2}$ ) of the instrumental noise in Advanced LIGO. 
We also consider a provisional noise curve for the Einstein Telescope [48]. We consider only a single detector of each type, rather than a combination of detectors, for a preliminary estimate of detectability.

Given two signals $h_{1}$ and $h_{2}$, define the usual inner product for a given noise spectrum $S_{h}(f)$ [49]:

$$
\left(h_{1} \mid h_{2}\right)=4 \operatorname{Re} \int_{0}^{\infty} \frac{\tilde{h}_{1}(f) \tilde{h}_{2}^{*}(f)}{S_{h}(f)} d f .
$$

This inner product yields a natural metric on a space of waveforms with distance between waveforms weighted by the inverse of the noise. We filter the detector output $s$ against an expected waveform $h$ using $(s \mid h)$. Then

$$
\varrho=\frac{(s \mid h)}{\sqrt{(h \mid h)}}
$$

is the optimal statistic to detect a waveform of known form $h$ in the signal $s$. If the detector output contains a particular waveform $h$ that is exactly matched by the template used, then the expectation value of $\varrho$ is the expected signal-to-noise ratio or SNR of that waveform:

$$
\bar{\varrho}=\sqrt{(h \mid h)} .
$$

Given a signal $h_{1}$ to be used as a template, one can consider whether a known departure from this signal can be measured. Assuming the modified waveform $h_{2}$ is known, the expected SNR of $h_{2}-h_{1}$ is similarly

$$
\overline{\varrho_{\text {diff }}}=\sqrt{\left(h_{2}-h_{1} \mid h_{2}-h_{1}\right)} .
$$

The two signals will be (marginally) distinguishable ${ }^{3}$. if the difference between the waveforms has $\overline{\varrho_{\text {diff }}} \geq 1$.

We analyse the measurability of the differences between hybrid inspiral-only waveforms matched to point particle PN waveforms in the early inspiral. After the numerical waveforms have been matched to the same post-Newtonian pointparticle inspiral, the signals will be aligned in time and phase. We can then then compare the resulting waveforms to each other, and to a PN-only waveform, using different Advanced LIGO noise spectra.

We report results in terms of the SNR measured by a single detector at an effective distance $D_{\text {eff }}=100 \mathrm{Mpc}$. Results can be scaled to any distance; as each measured $h \propto 1 / D, \overline{\varrho_{\text {diff }}} \propto$ $1 / D$.

The difference between waveforms due to finite size effects is not detectable in the NS-NS detection optimized configuration of Advanced LIGO for $\sim 100 \mathrm{Mpc}$ effective distances. However, in both narrowband and broadband the differences can be significant, and waveforms are distinguishable from each other and from the PP waveform. Note that this implies that even if the details of a NS-NS late inspiral signal are not known, the difference between it and a point particle

\footnotetext{
${ }^{3}$ Compare discussion in [50] of indistinguishability.
}

TABLE III: $\overline{\varrho_{\text {diff }}}$ in standard (NS-NS detection optimized) noise $\times\left(100 \mathrm{Mpc} / D_{\text {eff }}\right)$

\begin{tabular}{cccccc}
\hline Model & 2B & B & HB & H & 2H \\
\hline \hline PP & 0.32 & 0.45 & 0.55 & 0.46 & 0.69 \\
2B & 0 & 0.36 & 0.48 & 0.38 & 0.63 \\
B & & 0 & 0.21 & 0.12 & 0.58 \\
HB & & & 0 & 0.27 & 0.60 \\
H & & & & 0 & 0.58 \\
\hline
\end{tabular}

TABLE IV: $\overline{\varrho_{\text {diff }}}$ in broadband (burst-optimized) noise $\times\left(100 \mathrm{Mpc} / D_{\text {eff }}\right)$

\begin{tabular}{cccccc}
\hline Model & 2B & B & HB & H & 2H \\
\hline \hline PP & 1.86 & 2.32 & 2.67 & 2.38 & 2.89 \\
2B & 0 & 1.92 & 2.32 & 2.03 & 2.54 \\
B & & 0 & 0.81 & 0.80 & 2.27 \\
HB & & & 0 & 1.28 & 2.37 \\
H & & & & 0 & 2.35 \\
\hline
\end{tabular}

waveform should be measurable. The quantity $\overline{\varrho_{\text {diff }}}$ between the observed waveform and a best fit point particle waveform, limited to differences at high frequency, may be useful in itself to constrain possible EOS without reference to waveform details.

\section{A. Parameter estimation}

We will assume that EOS effects on the waveform impact the late inspiral only. For simplicity, we assume that orbital parameters, such as $M_{\text {tot }}$, mass ratio $\eta$, point particle postNewtonian coalescence time $t_{\mathrm{c}}^{\mathrm{PP}}$, and phase shift $\phi_{\mathrm{c}}^{\mathrm{PP}}$, are determined from the observations of the earlier inspiral waveform, with sufficient accuracy that their measurement uncertainty will not affect the accuracy to which the late inspiral effects determine the EOS parameters. These measurements would be made by a broad-band instrument, in which the signal-to-noise ratio is expected to be high $(\sim 40$ at $100 \mathrm{Mpc})$ and measurement accuracy is expected to be good [49]. Inaccuracies in these measurement could lead to biases in the measured EOS. This will be an important aspect to assess when high-quality binary neutron star simulations with various masses become abundant.

With a one-parameter family of waveforms sampled, we can estimate the accuracy to which this parameter can be measured. There are also other EOS-related parameters which are not considered. In the direct analysis of the measurability of the EOS parameter $p_{1}$, we ignore variations of $\Gamma$ within the core. Correspondingly, in the analysis of radius measurement, variations of the internal structures are neglected. Expanding coverage of the EOS parameter space is an ongoing project. However, these initial parameter choices are expected to give 
TABLE V: $\overline{\varrho_{\text {diff }}}$ in narrowband $1150 \mathrm{~Hz}$ noise $\times\left(100 \mathrm{Mpc} / D_{\text {eff }}\right)$

\begin{tabular}{cccccc}
\hline Model & 2B & B & HB & H & 2H \\
\hline \hline PP & 0.91 & 2.75 & 3.69 & 2.65 & 2.12 \\
2B & 0 & 1.92 & 2.92 & 1.82 & 1.45 \\
B & & 0 & 1.14 & 0.22 & 1.43 \\
HB & & & 0 & 1.34 & 2.25 \\
H & & & & 0 & 1.42 \\
\hline
\end{tabular}

TABLE VI: $\delta R(\mathrm{~km})$ in broadband (burst-optimized) noise $\times\left(D_{\text {eff }} / 100 \mathrm{Mpc}\right)$

\begin{tabular}{ccccc}
\hline Model & $2 \mathrm{~B}$ & $\mathrm{~B}$ & $\mathrm{H}$ & $2 \mathrm{H}$ \\
\hline \hline 2B & - & 0.63 & 1.28 & 2.17 \\
B & & - & 1.74 & 1.89 \\
$\mathrm{H}$ & & & - & 1.23 \\
\hline
\end{tabular}

the dominant contributions to finite size effects of the waveform ${ }^{4}$.

We estimate errors in parameter estimation to first order in $1 / \bar{\varrho}$ or, equivalently, in $\delta \theta^{A}$, using the Fisher matrix $\Gamma_{A B}=$ $\left(\partial_{A} h \mid \partial_{B} h\right)$ [49]. Its inverse, $\left(\Gamma^{-1}\right)^{A B}$, yields

$$
\overline{\delta \theta^{A} \delta \theta^{B}}=\left(\Gamma^{-1}\right)^{A B}
$$

so that the expected error in a given parameter $\theta^{A}$ is

$$
\overline{\left(\delta \theta^{A}\right)^{2}}=\left(\Gamma^{-1}\right)^{A A}
$$

and the cross terms of the inverse Fisher matrix yield correlations between different parameters.

With a few simulations of varying parameter value, we estimate $\partial h / \partial p_{1}$ and $\partial h / \partial R$ from two of the sampled waveforms, $h_{1}$ and $h_{2}$. For a single parameter $\theta$ (which can be taken to be either $p_{1}$ or $R$ ), we have

$$
\left.\frac{\partial h}{\partial \theta}\right|_{\theta=\left(\theta_{1}+\theta_{2}\right) / 2} \simeq \frac{h_{2}-h_{1}}{\theta_{2}-\theta_{1}}
$$

where $h_{1}=h\left(\theta_{1}\right)$ and $h_{2}=h\left(\theta_{2}\right)$, and then, for our oneparameter family where we neglect correlations with other parameters, we have to first order

$$
\overline{(\delta \theta)^{2}} \simeq \frac{\left(\theta_{2}-\theta_{1}\right)^{2}}{\left(h_{2}-h_{1} \mid h_{2}-h_{1}\right)} .
$$

Using adjacent pairs of models to estimate waveform dependence at an average parameter value, we then find estimates of radius measurability as shown in Table $\mathrm{VI}$ and $p_{1}$

\footnotetext{
4 For example, using the 1PN tidal effect estimates of [3 51] yields about $10 \%$ variation in the tidal terms contributing to binding energy and luminosity from changing internal structure-varying the apsidal constantwhile keeping radius fixed.
}

TABLE VII: $\delta \log \left(p_{1}\right)$, where $p_{1}$ is measured in dyn $\mathrm{cm}^{-2}$, in broadband (burst-optimized) noise $\times\left(D_{\text {eff }} / 100 \mathrm{Mpc}\right)$. Compare the range

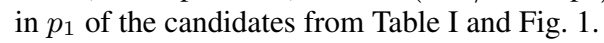

\begin{tabular}{ccccc}
\hline Model & 2B & B & H & 2H \\
\hline \hline 2B & - & 0.10 & 0.20 & 0.32 \\
B & & - & 0.25 & 0.26 \\
H & & & - & 0.17 \\
\hline
\end{tabular}

measurability as shown in Table VII for the burst-optimized noise configuration.

\section{B. Some sources of error}

We have cavalierly neglected many higher order but likely relevant effects in this preliminary analysis.

It is possible that tidal effects measurably influence the orbital evolution before the start of the numerical simulations, as estimated in [3], slowly enough not to be seen over the few cycles of the waveform matched to PP in this analysis. In one sense this analysis is a worst-case scenario, as it assumes exact PP behavior before the numerical match. Earlier drift away from point particle dynamics would give larger differences between waveforms, and more sensitive radius measurement, but poses a challenge by requiring accurate numerical simulation over many cycles to verify EOS effects. Combining numerical estimation with PN analyses like those of [3] and/or quasiequilibrium sequence information may clarify the transition between effectively PP and tidally influenced regimes.

The waveforms themselves have some residual eccentricity from initial data and finite numerical resolution. One can estimate eccentricity error by comparing the plus polarization to the cross polarization shifted by $\pi / 2$ from the same numerical waveform. This results in $\overline{\varrho_{\text {diff }}}$ of $\sim 0.3$ for $\mathrm{HB}$ and $2 \mathrm{H}$, rather than the expected quadrupole polarization cross-correlation of zero. (The other waveforms produce $\overline{\varrho_{\text {diff }}} \sim 0.05$ between polarizations).

The length of the inspirals, as discussed in the section on PN matching, limits precision in choosing the best match time. We can estimate these effects on current results by varying the match region considered; this changes $\overline{\varrho_{\text {diff }}}$ results by up to $\sim 0.5$ at $100 \mathrm{Mpc}$ in the broadband detector. The resolution from existing numerical simulations is thus comparable to the difference between parameters of the three closest models.

We have only a coarsely sampled family of waveforms; estimates of $\partial h / \partial \theta$ are limited by this. The value of $\overline{\varrho_{\text {diff }}}$ for HB-B and H-HB should be half that of H-B, but instead they are the same or greater-we are hitting the limit of numerical and matching accuracy. We can also estimate the validity of the linear parameter dependence in the central models by comparing $\mathrm{HB}$ to $(\mathrm{H}+\mathrm{B}) / 2$. This results in $\overline{\varrho_{\text {diff }}} \simeq 0.8$, another estimate of systematic error.

We conclude that, although these are of course first esti- 
mates, they should be better than order-of-magnitude; the uncertainty in each $\overline{\varrho_{\text {diff }}}$ is comparable to the $\overline{\varrho_{\text {diff }}}$ between the $\mathrm{H} / \mathrm{HB} / \mathrm{B}$ waveforms, and smaller than the $\overline{\varrho_{\text {diff }}}$ for larger differences in EOS and for comparison with PP.

Finally, we note that use of a Fisher matrix estimate of parameter measurement accuracy is fully valid only in high SNR limit of $\overline{\varrho_{\text {diff }}}>10$ [52], i.e., for distances $\lesssim 20 \mathrm{Mpc}$ in the broadband detector. The results do not take into account multiple detectors, nor multiple observations, nor parameter correlations. A full estimation of EOS parameter measurability will require more detailed analysis, with a larger set of inspiral simulations sampling a broader region of parameter space, e.g., with mass ratios departing from unity, and with several more orbits prior to merger.

\section{CONCLUSIONS}

Gravitational wave astrophysics provides a promising new window on behavior of cold dense matter. We estimate that realistic EOS will lead to gravitational inspiral waveforms which are distinguishable from point particle inspirals at an effective distance of $100 \mathrm{Mpc}$ or less in a burst-optimized Advanced LIGO configuration, as good or better than in narrow band detector configuration.

While the standard noise configuration of Advanced LIGO is not sensitive to the differences in the waveform, the preliminary standard noise curve of the Einstein Telescope indicate the ability to differentiate between EOS at roughly double the distance as broadband Advanced LIGO. In general, detuning detectors to be more sensitive at frequencies above $700 \mathrm{~Hz}$ will lead to improved gravitational wave constraints on neutron star EOS and radius.

First estimates of parameter measurability in broadband Advanced LIGO show $\delta R \sim 1 \mathrm{~km} \times\left(100 \mathrm{Mpc} / D_{\text {eff }}\right)$. One can also consider a direct constraint on the EOS pressure parameter $p_{1}$ at a rest mass density $\rho_{1}=5 \times 10^{14} \mathrm{~g} \mathrm{~cm}^{-3}$ of $\delta p_{1} \sim 10^{32} \mathrm{dyn} \mathrm{cm}^{-2}$ at an effective distance $D_{\text {eff }}=$ $100 \mathrm{Mpc}$. These estimates neglect correlations between these parameters and other details of internal structure, but such details are expected to give relatively small corrections to the tidal effects.

While our results must still be considered preliminary, they strongly motivate further work on gravitational wave constraints from binary neutron star inspirals. Future numerical simulations with longer inspirals and increased coverage of parameter space should improve the accuracy of the estimates.

\section{Acknowledgments}

This work was supported in part by NSF grants PHY0503366, PHY-0701817 and PHY-0200852, by NASA grant ATP03-0001-0027, and by JSPS Grants-in-Aid for Scientific Research(C) 20540275 and 19540263. CM thanks the Greek State Scholarship Foundation for support. Computation was done in part in the NAOJ and YITP systems.
[1] C. S. Kochanek, Astrophys. J. 398, 234 (1992).

[2] L. Bildsten and C. Cutler, Astrophys. J. 400, 175 (1992).

[3] É. É. Flanagan and T. Hinderer, Phys. Rev. D 77, 021502 (2008), 0709.1915.

[4] D. Lai and A. G. Wiseman, Phys. Rev. D 54, 3958 (1996), gr-qc/9609014.

[5] X. Zhuge, J. M. Centrella, and S. L. W. McMillan, Phys. Rev. D 54, 7261 (1996), arXiv:gr-qc/9610039.

[6] F. A. Rasio and S. L. Shapiro, Classical and Quantum Gravity 16, 1 (1999), arXiv:gr-qc/9902019.

[7] K. Uryu, M. Shibata, and Y. Eriguchi, Phys. Rev. D62, 104015 (2000), gr-qc/0007042.

[8] J. A. Faber, P. Grandclément, F. A. Rasio, and K. Taniguchi, Physical Review Letters 89, 231102 (2002), astro-ph/0204397.

[9] J. A. Faber, P. Grandclément, and F. A. Rasio, Phys. Rev. D 69, 124036 (2004), arXiv:gr-qc/0312097.

[10] M. Bejger, D. Gondek-Rosińska, E. Gourgoulhon, P. Haensel, K. Taniguchi, and J. L. Zdunik, Astronomy and Astrophysics 431, 297 (2005), astro-ph/0406234.

[11] D. Gondek-Rosińska, M. Bejger, T. Bulik, E. Gourgoulhon, P. Haensel, F. Limousin, K. Taniguchi, and L. Zdunik, Advances in Space Research 39, 271 (2007).

[12] M. Shibata, K. Taniguchi, and K. Uryū, Phys. Rev. D 71, 084021 (2005), arXiv:gr-qc/0503119.

[13] M. Shibata and K. Uryū, Prog. Theor. Phys. 107, 265 (2003).

[14] R. Oechslin and H.-T. Janka, Physical Review Letters 99, 121102 (2007).

[15] S. A. Hughes, Physical Review D 66, 102001 (2002), gr- qc/0209012.

[16] K. Taniguchi and E. Gourgoulhon, Phys. Rev. D68, 124025 (2003), gr-qc/0309045.

[17] T. Baumgarte, P. R. Brady, J. D. E. Creighton, L. Lehner, F. Pretorius, and R. Devoe, Phys. Rev. D 77, 084009 (2008).

[18] M. Shibata, Phys. Rev. D 60, 104052 (1999), arXiv:grqc/9908027.

[19] M. Shibata and K. Uryū, Phys. Rev. D 61, 064001 (2000), arXiv:gr-qc/9911058.

[20] M. Shibata and K. Taniguchi, Phys. Rev. D 73, 064027 (2006), arXiv:astro-ph/0603145.

[21] M. Shibata, K. Taniguchi, and K. Uryū, Phys. Rev. D 68, 084020 (2003), arXiv:gr-qc/0310030.

[22] M. Miller, P. Gressman, and W.-M. Suen, Phys. Rev. D 69, 064026 (2004), arXiv:gr-qc/0312030.

[23] P. Marronetti, M. D. Duez, S. L. Shapiro, and T. W. Baumgarte, Physical Review Letters 92, 141101 (2004), arXiv:grqc/0312036.

[24] Y. T. Liu, S. L. Shapiro, Z. B. Etienne, and K. Taniguchi, Phys. Rev. D 78, 024012 (2008), 0803.4193.

[25] T. Yamamoto, M. Shibata, and K. Taniguchi, Phys. Rev. D 78, 064054 (2008), 0806.4007.

[26] L. Baiotti, B. Giacomazzo, and L. Rezzolla (2008), 0804.0594[gr-qc].

[27] J. S. Read, B. Lackey, J. L. Friedman, and B. Owen, astro$\mathrm{ph} / 0812.2163$.

[28] J. M. Lattimer and M. Prakash, Astrophys. J. 550, 426 (2001), astro-ph/0002232. 
[29] S. E. Thorsett and D. Chakrabarty, Astrophys. J. 512, 288 (1999), arXiv:astro-ph/9803260.

[30] I. H. Stairs, Science 304, 547 (2004).

[31] K. Uryu and Y. Eriguchi, Phys. Rev. D61, 124023 (2000), grqc/9908059.

[32] S. Bonazzola, E. Gourgoulhon, and J.-A. Marck, Phys. Rev. Lett. 82, 892 (1999), gr-qc/9810072.

[33] E. Gourgoulhon, P. Grandclement, K. Taniguchi, J.-A. Marck, and S. Bonazzola, Phys. Rev. D63, 064029 (2001), grqc/0007028.

[34] K. Taniguchi and E. Gourgoulhon, Phys. Rev. D66, 104019 (2002), gr-qc/0207098.

[35] J. A. Isenberg, Int. J. Mod. Phys. D17, 265 (2008), grqc/0702113.

[36] J. R. Wilson and G. J. Mathews, Physical Review Letters 75, 4161 (1995).

[37] M. Shibata and T. Nakamura, Phys. Rev. D 52, 5428 (1995).

[38] T. W. Baumgarte and S. L. Shapiro, Phys. Rev. D 59, 024007 (1999), arXiv:gr-qc/9810065.

[39] M. Shibata and K. Taniguchi, Phys. Rev. D 77, 084015 (2008), 0711.1410.

[40] M. Campanelli, C. O. Lousto, P. Marronetti, and Y. Zlochower, Physical Review Letters 96, 111101 (2006), arXiv:grqc/0511048.
[41] J. G. Baker, J. Centrella, D.-I. Choi, M. Koppitz, and J. van Meter, Physical Review Letters 96, 111102 (2006), arXiv:grqc/0511103.

[42] A. Kurganov and E. Tadmor, J. Comput. Phys. 160, 241 (2000).

[43] M. Shibata, Phys. Rev. D 67, 024033 (2003).

[44] M. Boyle, D. A. Brown, L. E. Kidder, A. H. Mroué, H. P. Pfeiffer, M. A. Scheel, G. B. Cook, and S. A. Teukolsky, Phys. Rev. D 76, 124038 (2007), arXiv:0710.0158.

[45] A. Gopakumar, M. Hannam, S. Husa, and B. Brügmann, ArXiv e-prints 712 (2007), 0712.3737.

[46] L. E. Kidder, Physical Review D (Particles, Fields, Gravitation, and Cosmology) 77, 044016 (2008).

[47] B. Allen, W. G. Anderson, P. R. Brady, D. A. Brown, and J. D. E. Creighton (2005), gr-qc/0509116.

[48] C. Van Den Broeck, Tech. Rep. (2008), URL https: //workarea.et-gw.eu/et/WG4-Astrophysics / base-sensitivity/

[49] C. Cutler and É. E. Flanagan, Phys. Rev. D 49, 2658 (1994), gr-qc/9402014.

[50] L. Lindblom, B. J. Owen, and D. A. Brown (2008), 0809.3844.

[51] T. Hinderer, Astrophys. J. 677, 1216 (2008), 0711.2420.

[52] M. Vallisneri, Physical Review D (Particles, Fields, Gravitation, and Cosmology) 77, 042001 (2008). 